\title{
Connecting lysosomes and mitochondria - a novel role for lipid metabolism in cancer cell death
}

\author{
Karin Bartel ${ }^{1^{*}}$ D, Helmut Pein ${ }^{2}$, Bastian Popper ${ }^{3}$, Sabine Schmitt ${ }^{4}$, Sudha Janaki-Raman ${ }^{5}$, Almut Schulze ${ }^{5}$, \\ Florian Lengauer ${ }^{1}$, Andreas Koeberle ${ }^{2}$, Oliver Werz ${ }^{2}$, Hans Zischka ${ }^{4,6}$, Rolf Müller ${ }^{7}$, Angelika M. Vollmar ${ }^{1}$ and \\ Karin von Schwarzenberg ${ }^{1 *}$
}

\begin{abstract}
Background: The understanding of lysosomes has been expanded in recent research way beyond their view as cellular trash can. Lysosomes are pivotal in regulating metabolism, endocytosis and autophagy and are implicated in cancer. Recently it was discovered that the lysosomal V-ATPase, which is known to induce apoptosis, interferes with lipid metabolism in cancer, yet the interplay between these organelles is poorly understood.

Methods: LC-MS/MS analysis was performed to investigate lipid distribution in cells. Cell survival and signaling pathways were analyzed by means of cell biological methods (qPCR, Western Blot, flow cytometry, CellTiter-Blue). Mitochondrial structure was analyzed by confocal imaging and electron microscopy, their function was determined by flow cytometry and seahorse measurements.

Results: Our data reveal that interfering with lysosomal function changes composition and subcellular localization of triacylglycerids accompanied by an upregulation of PGC1a and PPARa expression, master regulators of energy and lipid metabolism. Furthermore, cardiolipin content is reduced driving mitochondria into fission, accompanied by a loss of membrane potential and reduction in oxidative capacity, which leads to a deregulation in cellular ROS and induction of mitochondria-driven apoptosis. Additionally, cells undergo a metabolic shift to glutamine dependency, correlated with the fission phenotype and sensitivity to lysosomal inhibition, most prominent in Ras mutated cells.
\end{abstract}

Conclusion: This study sheds mechanistic light on a largely uninvestigated triangle between lysosomes, lipid metabolism and mitochondrial function. Insight into this organelle crosstalk increases our understanding of mitochondria-driven cell death. Our findings furthermore provide a first hint on a connection of Ras pathway mutations and sensitivity towards lysosomal inhibitors.

Keywords: Lysosome, V-ATPase, Mitochondria, Fission, Apoptosis, Lipid metabolism, Cardiolipin

\section{Background}

Historically the lysosome has simply been regarded as the recycling compartment of a cell, yet recent research identified the lysosome as pivotal in regulating cellular metabolism [1]. Lysosomes are small organelles with an acidic interior, which host a large number of hydrolytic enzymes like proteases, lipases and nucleases. These

\footnotetext{
*Correspondence: karin.bartel@cup.uni-muenchen.de;

karin.von.schwarzenberg@cup.uni-muenchen.de

${ }^{1}$ Department of Pharmacy, Pharmaceutical Biology,

Ludwig-Maximilians-Universität München, Butenandtstr. 5-13, 81377 Munich, Germany

Full list of author information is available at the end of the article
}

hydrolases are responsible for degradation and recycling of macromolecules or even whole organelles thereby regulating endocytosis and autophagy [2]. Lysosome function or malfunction was found to play an important role in different diseases including cancer [3]. Interestingly, tumor cells often have an increased lysosomal activity and autophagy level as compared to non-malignant cells strengthening the hypothesis of the importance of lysosomes in resisting energetic stress conditions [4]. Especially the recent discovery of its key role in lipid metabolism highlights it as a promising organelle in regard of cancer treatment.

(c) The Author(s). 2019 Open Access This article is distributed under the terms of the Creative Commons Attribution 4.0 International License (http://creativecommons.org/licenses/by/4.0/), which permits unrestricted use, distribution, and reproduction in any medium, provided you give appropriate credit to the original author(s) and the source, provide a link to the Creative Commons license, and indicate if changes were made. The Creative Commons Public Domain Dedication waiver (http://creativecommons.org/publicdomain/zero/1.0/) applies to the data made available in this article, unless otherwise stated. 
Cellular lipid and cholesterol metabolism has emerged as novel target for cancer treatment, as it is frequently deregulated in tumor tissue. Yet, mechanistic data on how influencing lipid metabolism limits cancer cell survival is still limited [5,6]. While there are many descriptive studies showing alterations in cancer cell lipid metabolism as compared to healthy tissue and several studies link deregulation in lipid metabolism to malignancy, the influence of specific alterations on cellular organelles has not been thoroughly investigated $[7,8]$. Recently, we were able to identify a crucial role of the lysosome in cholesterol metabolism. Targeting the lysosomal V-ATPase, a proton pump necessary for lysosomal acidification, has been shown to restrict intracellular cholesterol availability and thereby inhibits tumor progression, linking lipid metabolism to lysosomes [9]. Targeting the V-ATPase with various natural compounds, such as archazolid, bafilomycin, concanamycin or iejimalide, has also been discovered to induce mitochondria-driven apoptosis in different cancer cells and to modulate autophagy, however the detailed mechanisms leading to mitochondrial apoptosis induction remain undiscovered [10-13].

The role of mitochondria in cell death induction has been studied extensively in the past. Their pivotal role in releasing proteins from the inter-membrane space to the cytosol and their power to activate caspases has long been known. Interestingly though interaction of mitochondria with other organelles has been less investigated [14-16]. In the present study we discovered a novel connection between lysosomes and mitochondria, in which cellular lipid metabolism plays an essential role. We provide a novel mechanistic insight into a triangle of lysosomes, mitochondria and lipid metabolism, shedding light on a missing link in lysosomal induced, mitochondria-driven cell death.

\section{Methods}

\section{Compounds and cell culture}

HUH7 were obtained from JCRB, BxPC3 and Panc03.27 were obtained from ATCC. STR profiling and routine testing for mycoplasma contamination were performed. HUH7 were grown in DMEM, 10\% FCS, BxPC3 were grown in RPMI-1640, 10\% FCS and Panc03.27 were grown in RPMI-1640, 15\% FCS, 10 Units/ml human recombinant insulin (PAN-Biotech $\mathrm{GmbH}$, Aidenbach, Germany \& Sigma-Aldrich). All cells were cultured under constant humidity at $37^{\circ} \mathrm{C}, 5 \% \mathrm{CO}_{2}$. For $\mathrm{HUH7}$, all plastic ware was pre-coated with $0.001 \%$ collagen G (PBS). Archazolid A was provided by Rolf Müller, Torin1, CCCP, BPTES, UK5099 and Etomoxir were purchased from Sigma-Aldrich, and dissolved in DMSO (Sigma-Aldrich).

\section{Triaclyglycerid (TAG) and acyl-CoA analysis}

HUH7, HepG2 or Hep3 cells were treated as indicated and collected by centrifugation. Lyososomes [17] or mitochondria were isolated as described previously [18], or whole cells were used. Cell pellets and subcellular fractions were frozen in liquid nitrogen and stored at $80{ }^{\circ} \mathrm{C}$ until use. TAGs were extracted using a mixture of PBS pH 7.4, methanol, chloroform, and saline (14:34:35: 17), separated on an AcquityTM UPLC BEH C8 column $(1.7 \mu \mathrm{m}, 2.1 \times 100 \mathrm{~mm}$, Waters, Milford, MA) using an AcquityTM Ultraperformance LC system (Waters) and detected by a QTRAP 5500 mass spectrometer (Sciex, Darmstadt, Germany) equipped with an electrospray ionization source as described [19]. Acyl-CoAs were extracted with methanol/water (70/30), separated on an AcquityTM UPLC BEH C18 column $(1.7 \mu \mathrm{M}, 2.1 \times 50$ $\mathrm{mm})$, and analyzed in the positive ion mode based on the neutral loss of $2^{\prime}$-phospho-ADP $([\mathrm{M}+\mathrm{H}-507]+)$ as previously reported for malonyl-CoA [20]. 1,2-Dimyristoyl-sn-glycero-3-phosphatidylcholine and [13C3]-malonyl-CoA were used as internal standards for TAGs and acyl-CoAs, respectively.

\section{Confocal microscopy}

30,000 cells/well were seeded on IBIDI $\mu$-slides (IBIDI, Martinsried, Germany) one day before treatment as indicated $(24 \mathrm{~h})$. For antibody staining, cells were washed (PBS), fixed (3\% Paraformaldehyde) for $30 \mathrm{~min}$, permeabilized (0.1\% Triton-X) and unspecific binding was blocked (5\% BSA) after treatment. Primary antibodies (Lamp3/sc-15363, Hsp60/sc-1052, Cox4/4844 NEB, ACADVL/ab118183, ACADM/ab118183, HADHA/ ab118183, TFE3/PA5-35210, MITF/sc-71588, TFEB/ A303-673A) were applied over night at $4{ }^{\circ} \mathrm{C}$, secondary antibodies (Alexa-Fluor 488/A-11008, Alexa-Fluor 546/ A-10040, Alexa-Fluor 633/A-21082) and Hoechst33342 for $45 \mathrm{~min}$ at $25^{\circ} \mathrm{C}$. LD were stained by $2 \mu \mathrm{M}$ Bodipy ${ }^{\mathrm{m}}$ 493/503 for $30 \mathrm{~min}$, fatty acids were tracked by adding $1 \mu \mathrm{M}$ BODIPY ${ }^{\text {mo }} 558 / 568 \mathrm{C}_{12}$ (both: Thermo Fisher) $16 \mathrm{~h}$ prior to end of treatment. Cells were washed, mounted with FluorSave ${ }^{\mathrm{Tx}}$ Reagent (Beckman Coulter) and covered with a glass coverslip. For life cell imaging $2 \mu \mathrm{M}$ Bodipy 493/503 was added $30 \mathrm{~min}$ prior to imaging, $1 \mu \mathrm{M}$ BODIPY ${ }^{\mathrm{mm}} 558 / 568 \mathrm{C}_{12}$ was added $16 \mathrm{~h}$ prior to imaging, 100 nM LysoTracker ${ }^{\text {tm }}$ Blue DND-22 or $100 \mathrm{nM}$ MitoTracker $^{\text {Tw }}$ Green FM were added for 30 min to visualize lysosomes or mitochondria. Medium was exchanged and images were acquired using a Leica TCS SP 8 SMD confocal microscope (Leica TCS SP 8 SMD, Wetzlar, Germany) with a top stage incubator (Oko Lab, Ottaviano, Italy).

\section{Flow cytometry}

For surface expression cells were treated as indicated $(24 \mathrm{~h})$, harvested and stained with specific antibody against CD 36 (sc-5522) and fluorescent secondary antibody Alexa-Fluor 546 (A-11056). Mitochondrial 
mass was detected after labelling mitochondria (MitoTracker $^{\text {ma }}$ Green FM) for 30 min prior to the end of treatment time. For analysis of mitochondrial membrane potential cells were loaded with DIOC6 (Sigma Aldrich) or JC-1 (Sigma Aldrich). Enzyme abundance of FAO enzymes was detected according to manufacturer's protocol (Abcam, ab118183). Mitochondrial superoxide and cellular ROS were detected by loading the cells with MitoSOX ${ }^{\mathrm{mw}}$ (M36008, Thermo Fisher) or CDCFDA (C1165, Thermo Fisher) prior to harvesting. Cytosolic cytochrome $\mathrm{C}$ was detected by specific antibody (NEB, 4272) and fluorescent secondary antibody Alexa-Fluor 546 (A-11056). Subdiploid DNA content was determined according to Nicoletti et al. [15]. Briefly, cells were treated as indicated, harvested, permeabilized with sodiumcitrate containing Triton X-100, stained with $25 \mu \mathrm{g} / \mathrm{ml}$ propidiumiodide and analyzed. Subdiploid cells left of the G1-peak were considered as apoptotic. All cells were analyzed by flow cytometry (Canto II, Beckton Dickinson, Heidelberg, Germany).

\section{Quantitative real-time PCR}

Total mRNA was isolated from cell culture samples according to manufacturer using Qiagen RNeasy Mini Kit (Qiagen, Hilden, Germany). For cDNA synthesis, the High Capacity cDNA Rerverse Transcription Kit (Applied Biosystems, Foster City, CA) was used. qRTPCR was performed with the QuantStudio ${ }^{\text {Tx }} 3$ System (Thermo Fisher) and the SYBR Green PCR Master Mix (Thermo Fisher) according to the manufacturer's instructions. All designed primers were purchased from Metabion (Martinsried, Germany).

\section{Western blot}

For preparation of whole cell lysate, cells were collected by centrifugation, washed with ice-cold PBS, and lysed for $30 \mathrm{~min}$ in $1 \%$ Triton $\mathrm{X}-100,137 \mathrm{mM} \mathrm{NaCl}$, and 20 $\mathrm{mM}$ Tris-Base ( $\mathrm{pH}$ 7.5) containing the protease inhibitor complete (Roche). Lysates were centrifuged at 10,000 g/ $10 \mathrm{~min}$ at $4{ }^{\circ} \mathrm{C}$. Mitochondria were isolated as described previously [10]. Equal amounts of protein were separated by SDS-PAGE and transferred to nitrocellulose membranes (Hybond-ECLTM, Amersham Bioscience). Membranes were blocked with 5\% BSA in PBS containing 0.1\% Tween 20 for $2 \mathrm{~h}$ and incubated with specific antibodies against PGC1 $\alpha /$ ab54481, PPAR $\alpha /$ MA1-822, Mitofusin-1/ 14739 NEB, Drp-1/8570 NEB, pDrp-1 Ser 637/6319 NEB, pDrp-1 Ser 616/4494 NEB, ACADVL/ab118183, ACADM/ab118183, HADHA/ab118183, TFE3/PA535210, MITF/sc-71588, TFEB/A303-673A, Bax/sc-493, $\mathrm{Bak} / \mathrm{ab} 32371$ ) over night at $4{ }^{\circ} \mathrm{C}$. Proteins were visualized by secondary antibodies conjugated to horseradish peroxidase (HRP) and freshly prepared ECL solution, containing $2.5 \mathrm{mM}$ luminol. Chemiluminescence signal was detected with the ChemiDoc ${ }^{\text {ru }}$ Touch Imaging System (Bio-Rad, Munich, Germany).

\section{Analysis of free fatty acids}

Free fatty acids were detected according to manufacturer's protocol (MAK044, Sigma Aldrich). Briefly, cells were treated as indicated, harvested and homogenized in $1 \%$ Triton X-100 in chloroform. Organic phase was collected after centrifugation and vacuum dried. Lipids were re-dissolved in assay buffer and incubated with reaction mix. Absorbance was measured with an infinite F200Pro plate reader (Tecan) and is proportional to free fatty acid content.

\section{Analysis of cardiolipins}

Detection of cardiolipins in cell lysates or in isolated mitochondria was performed according to manufacturer's protocol (\#K944-100, BioVision). Briefly, cells were treated as indicated, harvested and lysed. Lysate was loaded with a CL-Probe and incubated for $10 \mathrm{~min}$ at $25^{\circ} \mathrm{C}$. Probe fluorescence was recorded at Ex/Em 304/ $480 \mathrm{~nm}$ with an infinite F200Pro plate reader (Tecan) and is proportional to cardiolipin content.

\section{Electron microscopy}

Samples were fixed with $2.5 \%$ Glutaraldehyde in $0.1 \mathrm{M}$ Sodium Cacodylate Buffer, pH 7.4 for $24 \mathrm{~h}$ at the minimum. Glutaraldehyde was removed, samples were washed 3x with 0.1 M Sodium Cacodylate Buffer, $\mathrm{pH}$ 7.4. Postfixation and prestaining was done for $45-60 \mathrm{~min}$ with $1 \%$ osmium tetroxide, ddH2O, $3.4 \% \mathrm{NaCl}$ and $4.46 \%$ potassium dichromate $\mathrm{pH} 7.2$. Samples were washed $3 \mathrm{x}$ with $\mathrm{dd} \mathrm{H} 2 \mathrm{O}$ and dehydrated with an ascending ethanol series (15 min with 30, 50, 70, 90 and 96\%, respectively and $2 \times 10 \mathrm{~min}$ with $100 \%$ ) and propylene oxide $(2 \times 30 \mathrm{~min})$. Subsequently, samples were embedded in Epon (3.61 M Glycidether 100, 1.83 M Methylnadicanhydride, $0.92 \mathrm{M}$ Dodecenylsuccinic anhydride, 5.53 $\mathrm{mM}$ 2,4,6-Tris (dimethylaminomethyl)phenol. Ultrathin sections were sliced with an Ultramicrotome (Ultracut E; Reichert und Jung, Germany) and automatically stained with UranyLess EM Stain (Electron Microscopy Sciences) and 3\% lead citrate using the contrasting system Leica EM AC20 (Leica, Wetzlar, Germany). The samples were examined with an JEOL - 1200 EXII transmission electron microscope (JEOL GmbH, Freising, Germany). Buffers were purchased from Serva Electrophoresis $\mathrm{GmbH}$. Mitochondrial area and Feret diameter were analyzed identically for all samples using ImageJ.

\section{Seahorse}

Metabolic activity was analyzed using an Agilent Seahorse 96XF device and respective kits. Cell mito stress test was performed as described in the manufacturer's 
protocol (Kit 103015-100). Mitochondrial fuel dependency and capacity were determined according to manufacturer's protocol (Kit 103270-100). Briefly, cells were pre-treated, medium was exchanged for seahorse medium. Compounds were present during the entire measurement. Respiratory parameters, fuel dependency and capacity were calculated using the Seahorse Wave Desktop Software and the Seahorse XF Cell Mito Stress Test Report Generator or the Seahorse XF Mito Fuel Flex Test Report Generator (Agilent Technologies).

\section{NADPH/NADP+ measurements}

NADP+/NADPH levels were assessed using the NADP/ $\mathrm{NADPH}-\mathrm{Glo}^{\mathrm{Tm}}$ Assay according to manufacturer's protocol (Promega). Briefly, cells were treated as indicated (24 h). Media was replaced with PBS and basic lysis solution was added. Lysates were transferred to white walled 96-well plate and splitted for NADP+ and NADPH measurements. Respective solutions were added and NADP/ $\mathrm{NADPH}-\mathrm{Glo}^{\mathrm{Tm}}$ Detection Reagent was added. After 60 min incubation at $25^{\circ} \mathrm{C}$ luminescence was detected using an Orion II microplate luminometer (Berthold Detection Systems, Pforzheim, Germany).

\section{Proliferation}

Proliferation was assessed with the CellTiter-Blue ${ }^{\circ}$ Cell Viability Assay (Promega, Madison, WI, USA). 5,000 cells/well were seeded, basal metabolic activity was determined $(24 \mathrm{~h})$ and cells were treated as indicated for $72 \mathrm{~h}$. CellTiter-Blue ${ }^{\circ}$ Reagent was added for $4 \mathrm{~h}$ and the absorbance at $590 \mathrm{~nm}$ was measured in a Sunrise ELISA reader (Tecan, Maennerdorf, Austria) and is proportional to the cell number.

\section{Statistics}

Experiments have been performed at least three times, unless stated otherwise. For analysis representative images out of three independent experiments are shown. Bars are the mean + SEM of three independent experiments. $P$ values of $p^{*}<0.05$ (One-way ANOVA, Dunnett post test or student $\mathrm{t}$-test) were considered significant.

\section{Results}

Impaired lysosomal function changes cellular lipid profile We recently showed, that lysosomal malfunction leads to alterations in cholesterol metabolism and subsequently to impaired proliferation of cancer cells [9]. To decipher the role of the lysosome in lipid regulation of cancer cells we disrupted lysosomal function by treatment with archazolid (Arch). Archazolid is a potent inhibitor of the lysosomal VATPase, which causes a drastic increase in luminal $\mathrm{pH}$ and thereby disrupts lysosomal function. Arch has shown promising anti-cancer activity in various studies $[9,10,21-23]$. We treated different hepatocellular carcinoma (HCC) cell lines with Arch for $24 \mathrm{~h}$ and subsequently analyzed composition of triacylglycerid species (TAG). We found that composition of TAG is strongly changed upon V-ATPase inhibition (Fig. 1a) shifting a lipid profile with an increased degree of saturation, while total TAG content is barely affected (Additional file 1: Figure S1A). The relative abundance of different lipid species in the HCC cell lines was comparable containing predominantly TAG with monoand poly-unsaturated fatty acids (Additional file 1: Figure S1B-D). Furthermore, we were interested in the lipid composition of different organelles after Arch treatment. Hence, we isolated lysosomes and mitochondria of HUH7 cells after treatment and again analyzed TAG composition. In comparison to whole cells (Fig. 1a), TAG composition of lysosomes (Fig. 1b) was altered in the same manner, while palmitic acid containing TAGs were downregulated in mitochondria (Fig. 1c), total TAG content of isolated organelles did not change (Additional file 1: Figure S1E-F). Along the line, we also observed changes in Acyl-CoA levels after VATPase inhibition (Fig. 1d). Next, we investigated condition and content of lipid droplets (LD), the lipid storage organelles. In order to assess whether our observations are specific to V-ATPase inhibition or rather a general response to lysosomal stress, we included treatment with the mTOR inhibitor Torin 1 and starvation with HBSS, which have been shown to induce lysosomal stress and create a similar metabolic phenotype as compared to VATPase inhibition [24-26]. We observed that lysosomal stress in general leads to a change in LD size and distribution (Fig. 1e), as well as a decrease in overall LD content (Fig. 1f). Yet, localization of LD was varied between different stress conditions (Fig. 1E). Overall, we found that impairment of lysosomal function changes cellular lipid profile and subcellular localization of lipids.

\section{V-ATPase inhibition leads to alterations in lipid metabolism}

Alterations in lipid composition might in principle arise from changes in synthesis, uptake or degradation processes, which we analyzed one after another. A crucial regulator of lipid metabolism is PGC1 $\alpha$. PGC1 $\alpha$ is a master regulator of cellular energy metabolism, including mitochondrial beta oxidation, i.e. degradation of lipids to generate energy. Additionally, PGC1 $\alpha$ is controlling lipid metabolism by transcriptional regulation of PPAR $\alpha$, which promotes uptake, utilization, and catabolism of fatty acids. Interestingly, 4:0 Co-A, an intermediate of beta-oxidation was significantly increased after Arch treatment (Fig. 1d). Quantitative real-time PCR (qPCR) measurements revealed that inhibition of V-ATPase tremendously increases PGC1 $\alpha$ expression, while mTOR inhibition and starvation do not (Fig. 2a). Additionally, mRNA (Fig. 2b) and protein level (Fig. 2c) of PPAR $\alpha$ is upregulated upon V-ATPase 
A

\section{$+\operatorname{Arch} \mathrm{A}$

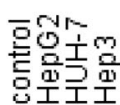

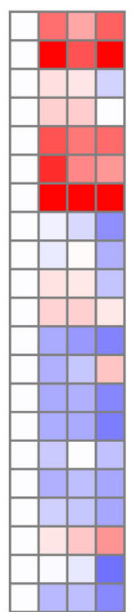

Annotation

16:0/16:0/16:0

16:0/16:0/18:0

16:0/16:0/16:1

$16: 0 / 16: 0 / 18: 1$

16:0/18:0/18:1

16:0/16:1/18:0

18:0/18:0/18:1

16:0/16:1/18:1

16:0/18:1/18:1

18:0/16:1/18:1

18:0/18:1/18:1

18:1/16:1/16:1

$18: 1 / 18: 1 / 18: 1$

16:0/18:1/18:2

18:0/16:1/18:2

16:0/18:0/20:3

18:0/18:1/20:3

16:0/18:1/20:4

16:0/16:0/22:6

16:0/18:1/22:6

18:0/18:2/22:6

0 100300 [\% control]

D
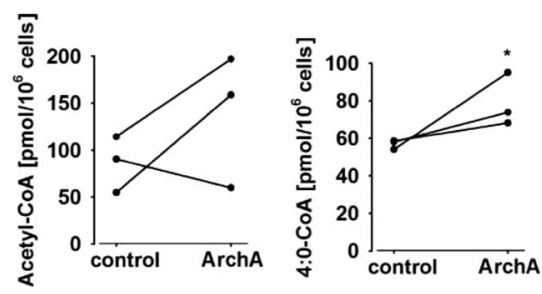

E

Control
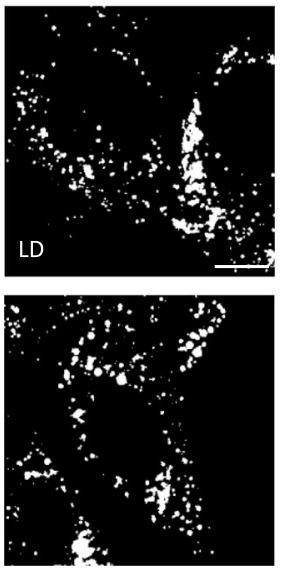

Torin 250nM
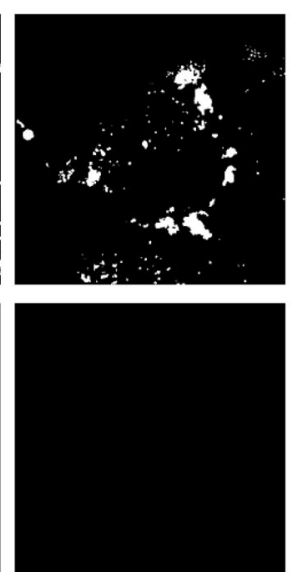

HBSS
B

爱突

Annotation

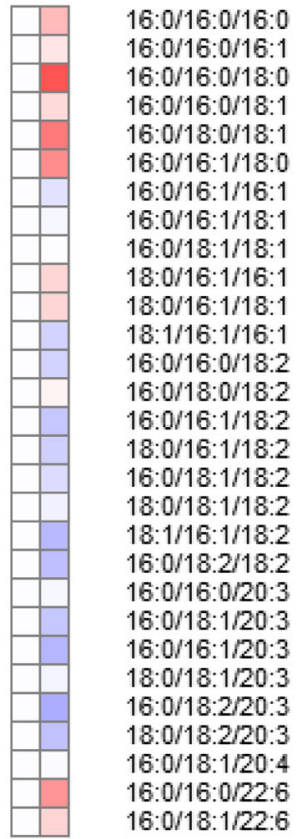
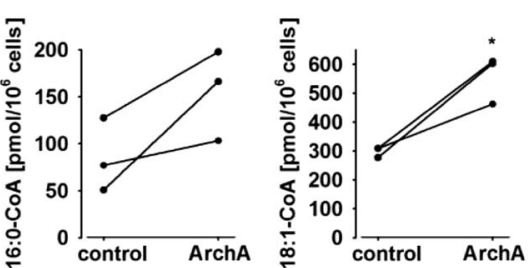

$F$
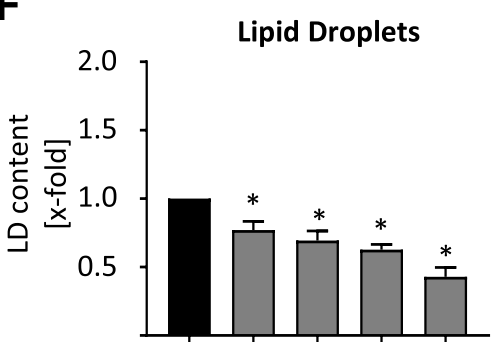

Arch $10 \mathrm{nM}$

Torin $250 \mathrm{nM}$

HBSS

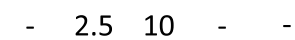

C 我突

Mitochondria

Annotation

16:0/16:0/16:0

16:0/16:0/16:1

16:0/16:0/18:0

16:0/16:0/18:1

16:0/18:0/18:1

18:0/18:0/18:1

16:0/16:1/18:0

16:0/16:1/16:1

16:0/16:1/18:1

16:0/18:1/18:1

18:0/16:1/16:1

18:0/16:1/18:1

18:1/16:1/16:1

16:0/16:0/18:2

$16: 0 / 18: 0 / 18: 2$

$16: 0 / 16: 1 / 182$

16:0/18:1/18:2

16:0/18:2/18:2

$18: 0 / 16: 1 / 18: 2$

18:0/18:1/18:2

18:0/18:2/18:2

$18: 1 / 16: 1 / 18: 2$

16:0/16:0/20:3

$16: 0 / 16: 1 / 20: 3$

$16 \cdot 0 / 18 \cdot 1 / 20 \cdot 3$

16:0/18:2/20:3

18:0/16:1/20:3

18:0/18:1/20:3

18:0/18:2/20:3

16:0/16:0/20:4

16:0/18:0/20:4

16:0/18:1/20:4

16:0/16:1/20:4

18:0/18:1/20:4

18:0/18:2/20:4

16:0/18:0/22:5

16:0/18:1/22:5

16:0/16:0/22:6

16:0/16:1/22:6

16:0/18:1/22:6

Fig. 1 (See legend on next page.) 
(See figure on previous page.)

Fig. 1 V-ATPase inhibition influences lipid profile. Cells were treated as indicated (24 h). Lipids from whole cells (HUH7, HepG2 and Hep3B) (a), lysosomes (HUH7) (b) or mitochondria (HUH7) (c) were isolated and TAG composition was analyzed by UPLC-MS/MS. Heatmaps display percentage increase (red) and decrease (blue) of respective TAG species compared to DMSO control. d Lipids from whole cells (HUH7) were isolated and cholesteryl ester composition was analyzed by mass spectrometry (student t-test). e, f Cells were loaded with Bodipy 493/503 to stain lipid droplets (LD). e LD size and localization was analyzed by confocal microscopy. Scale bar $10 \mu \mathrm{m}$. Representative images out of three independent experiments are shown. Bars are the mean + SEM of three independent experiments. $\mathbf{f} L D$ content was quantified by flow cytometry. $p^{*}<0.05$ (One-way ANOVA, Dunnett post test)

treatment. These data suggest that cells specifically upregulate catabolism of lipids upon treatment with Arch. Of note, other relevant downstream targets of PGC1 $\alpha$, namely NRF1, NRF2 and ERR $\alpha$ are not influenced in their expression upon induction of lysosomal stress (Additional file 2: Figure S2A-C). Furthermore, cells increase uptake of fatty acids as the surface expression of CD36, also called fatty acid translocase, is increased upon V-ATPase inhibition (Fig. 2d). Additionally, the level of free fatty acids, which are essential for energy generation in mitochondria are increased after Arch treatment (Fig. 2e). These findings strongly suggest that cells induce lipid degradation, plausibly to sustain energy generation upon V-ATPase inhibition. Free fatty acids can be converted to acetyl-CoA by mitochondria via $ß$-oxidation, feeding into the TCA cycle and fueling ATP synthesis via oxidative phosphorylation. For this, proper mitochondrial function is essential, in particular mitochondrial membrane composition is important, as the respiratory complexes are assembled there. As we observed specific alterations in TAG in mitochondria after treatment with Arch (Fig. 1c), we hypothesized that also cardiolipin content might be affected. Cardiolipins are a special lipid species representing essential mitochondrial inner-membrane lipids, essentially contributing to membrane curvature and therefore mitochondrial function [27]. We found that the content of cardiolipins is reduced in cells after V-ATPase inhibition (Fig. 2 F-G), indicating mitochondrial malfunction. Cells are furthermore able to facilitate lipids by degradation in lysosomes, a process called lipophagy. In order to determine lipid localization, we analyzed colocalization of a labelled C-12 lipid with lysosomes and mitochondria (Fig. 2h, Additional file 2: Figure S2D-E), yet no significant overlay could be detected, indicating that lipophagy is not predominant. However, confocal images indicate a shift of mitochondrial structure from long networks to short, round-shaped mitochondria (Cox4) (Fig. 2h) upon V-ATPase inhibition, indicating mitochondrial fission.

\section{Lysosomal mediated structural changes in mitochondria}

Mitochondrial fission is implicated in the cellular stress response and apoptosis and it usually precedes mitochondrial degradation by lysosomes, i.e. mitophagy [16, 28]. A co-staining of lysosomes and mitochondria showed no overlay after V-ATPase or mTOR inhibition, but after starvation, displaying an indication for mitophagy (Fig. 3a).
Flow cytometry furthermore revealed that the mitochondrial mass is not changed after V-ATPase inhibition (Fig. 3b), indicating that no significant degradation of mitochondria is induced. Western Blot analysis of Mitofusin-1 and Drp1 confirmed an increased phosphorylation of Drp1, confirming the fission phenotype, since phosphorylated Drp1 mediates the final step of fission, the separation to two daughter mitochondria (Fig. 3d, Additional file 3: Figure S3A). Detailed structural analysis of mitochondria in electron microscopy displayed rather elongated mitochondria in control cells and smaller, round-shaped mitochondria in Arch treated cells (Fig. 3d, Additional file 3: Figure S3B). This is also reflected after analyzing mitochondrial area in EM images (Fig. 3e) and Feret diameter (Fig. 3f). Hence, we hypothesize that cancer cells induce mitochondrial fission after V-ATPase inhibition in order to sustain sufficient energy production.

\section{Lysosomal stress disrupts mitochondrial function}

As we observed mitochondrial fission after V-ATPase inhibition, we checked whether mitochondria are still functional. Flow cytometric analysis of cells stained with DIOC6, a dye that is selectively localized to intact mitochondria, revealed that mitochondrial membrane potential is disrupted indicating a loss of mitochondrial function (Fig. 4a). A staining with JC1 confirmed this finding, by displaying a high amount of mitochondria with dissipated membrane potential (Fig. $4 \mathrm{~b}$ ). The effect of Arch on mitochondrial membrane potential was almost as prominent as carbonyl cyanide $\mathrm{m}$-chlorophenyl hydrazine (CCCP), a known uncoupling agent. To further characterize the state of mitochondrial function, we analyzed transcription, protein abundance and localization of important enzymes in fatty acid beta oxidation, namely ACADVL, ACADM and HADHA. These enzymes catalyze the first steps of fatty acid beta oxidation and differ in affinity towards fatty acids with different chain lengths. While ACADVL is specific for very long chain fatty acids, ACADM is specific for medium chain fatty acids and HADHA catalyzes three out of four steps of beta-oxidation of long chain fatty acids. After V-ATPase inhibition protein and expression level of ACADVL are increased. mTOR inhibition rather led to a decrease in enzyme abundance, while starvation showed no consistent alterations (Fig. 4c, d, Additional file 4: Figure S4A). Confocal imaging of 


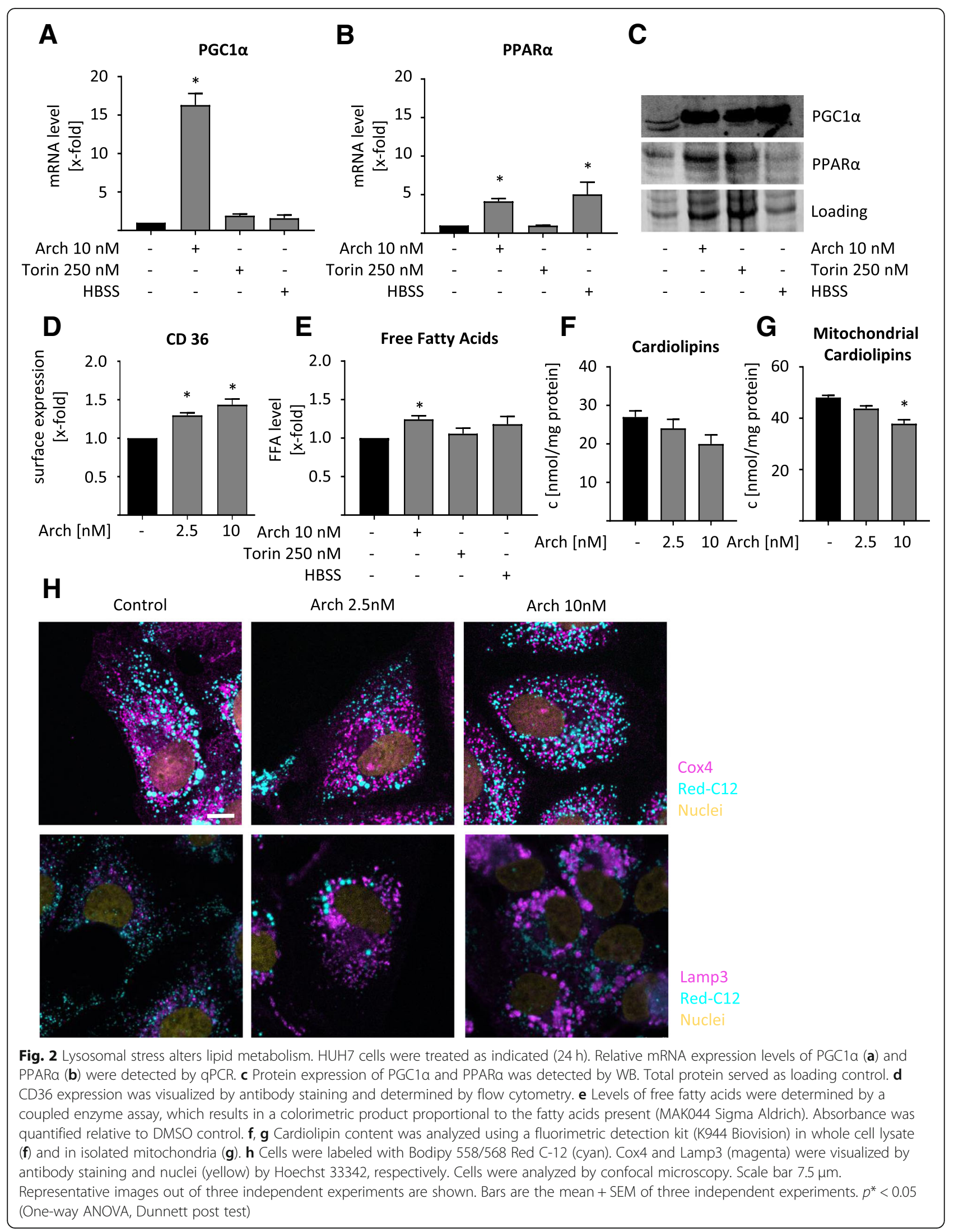




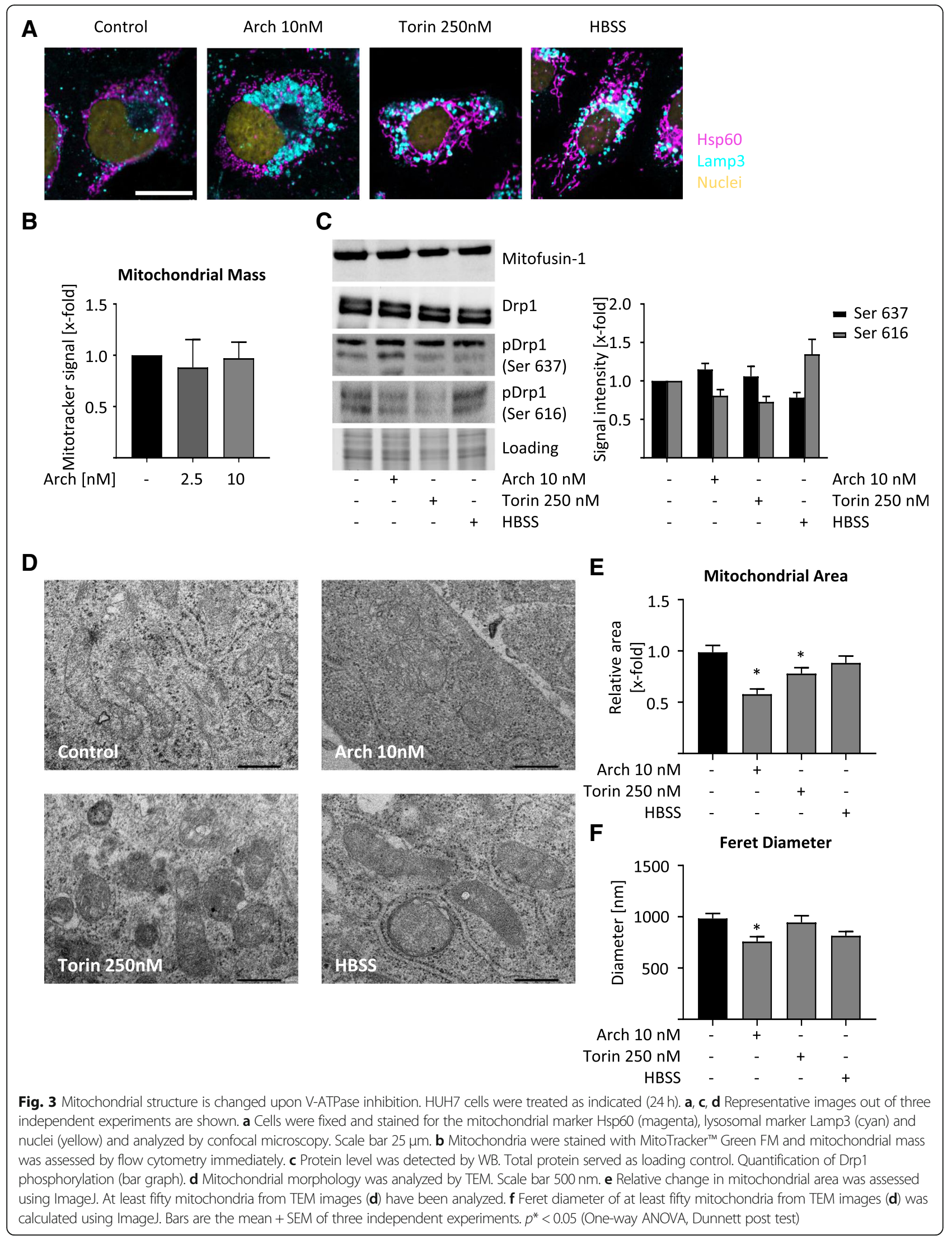


A

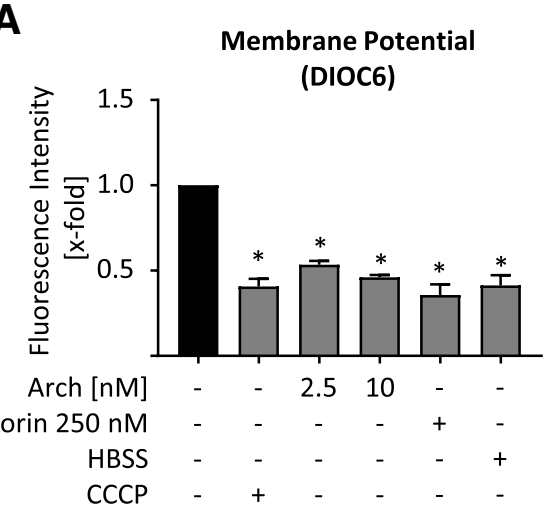

C

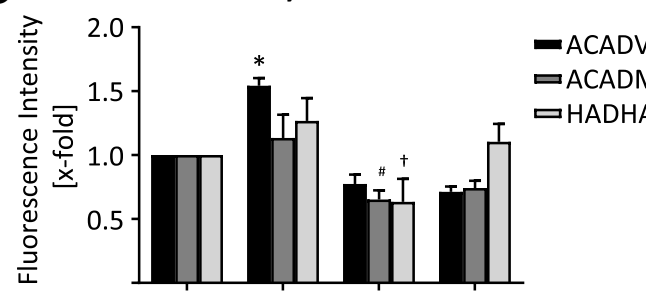

Arch $10 \mathrm{nM}$

Torin $250 \mathrm{nM}$

HBSS

E
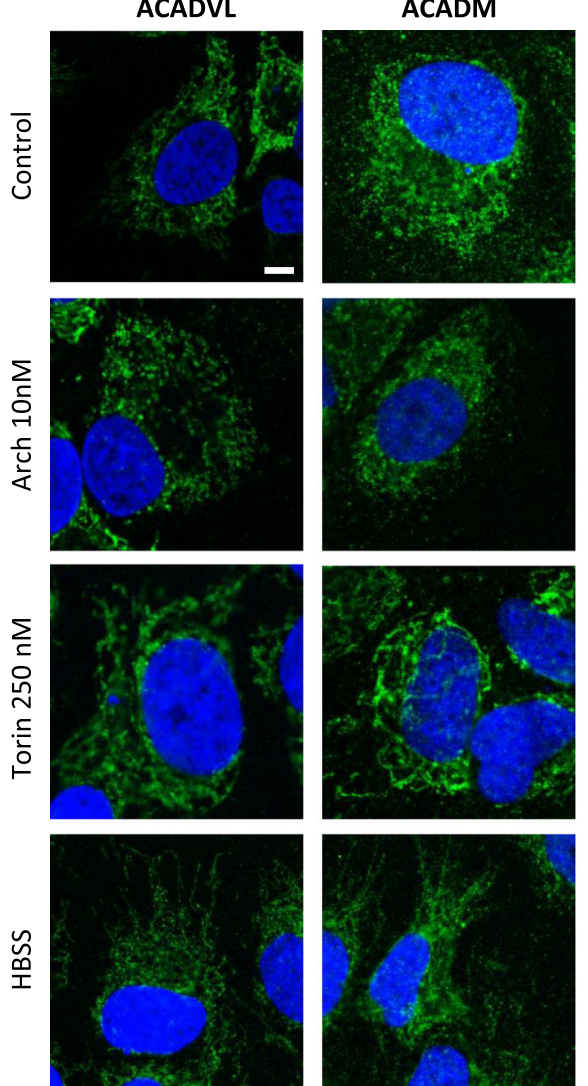

B

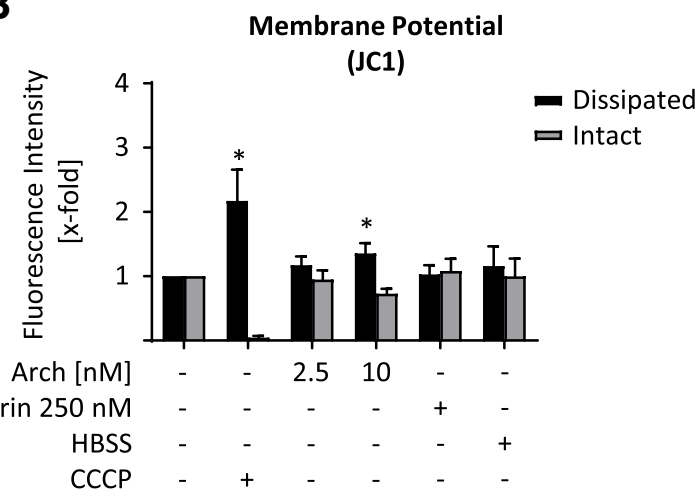

D

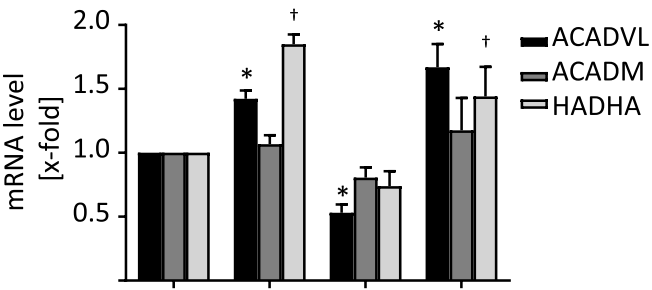

Arch $10 \mathrm{nM}$ Torin $250 \mathrm{nM}$

ADHA
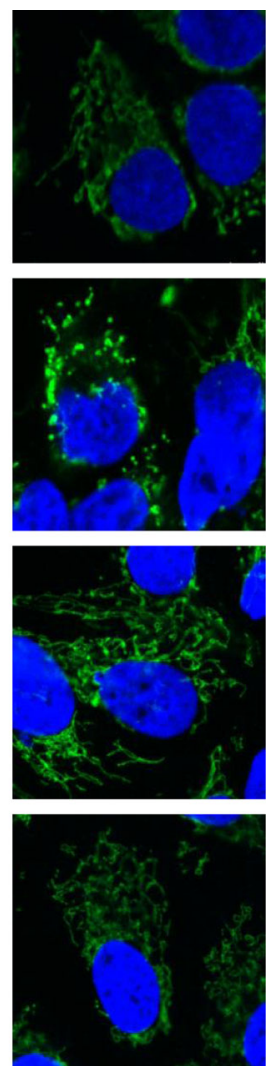

Enzyme Nuclei

Fig. 4 (See legend on next page.) 
(See figure on previous page.)

Fig. 4 Alterations in mitochondrial function due to lysosomal stress. HUH7 cells were treated as indicated (24 h). DIOC6 (a) or JC1 (b)

fluorescence were quantified by flow cytometry. c Enzyme abundance was quantified by flow cytometry. $\mathbf{d}$ Relative mRNA expression levels of ACADVL*, ACADM ${ }^{\#}$ and $\mathrm{HADHA}^{\dagger}$ were assessed by qPCR. a-d Bars are the SEM of three independent experiments. $p^{* \# \dagger}<0.05$ (One-way ANOVA Dunnett post test) (e) Cells were fixed and stained for ACADVL, ACADM and HADHA, respectively, (green) and nuclei (blue) and analyzed by confocal microscopy. Scale bar $7.5 \mu \mathrm{m}$. Representative images out of three independent experiments are shown

enzyme localization confirmed the Arch induced fission phenotype as observed before (Fig. 4e). These data indicate a reduction in mitochondrial function. To prove this impairment, we performed seahorse measurements facilitating a mitochondrial stress test. After pre-treatment of the cells with the respective compounds, oxygen consumption rate (OCR) was measured over time and inhibitors of mitochondrial function (oligomycin, FCCP and Rotenone+Antimycin) were added sequentially (Fig. 5a) in order to calculate respiratory parameters of the cells (Fig. 5b). After treatment with Arch the basal respiration, as well as maximum respiratory capacity and ATP production are strongly decreased compared to control cells (Fig. $5 \mathrm{a}, \mathrm{b})$, confirming an impairment in mitochondrial function. The reduction in ATP levels could also be confirmed in a CellTiter-Glo assay (Additional file 4: Figure S4B). Additionally, we used the mitochondria fuel flex test to investigate which source the cells mainly use for beta oxidation. This test blocks different fuels from being oxidized in the mitochondria by using the specific inhibitors BPTES, which inhibits conversion of glutamine to glutamate, UK50699, which inhibits mitochondrial pyruvate carrier, therefore blocking glucose as energy source and etomoxir, which inhibits palmitate uptake into mitochondria and therefore blocks fatty acid oxidation. We found that upon V-ATPase inhibition cells become more dependent on glutamine as energy source (Fig. $5 \mathrm{c}$ ), while the capacity to use each single energy source remains unchanged (Additional file 4: Figure S4C). Furthermore, we analyzed mitochondrial superoxide (SOX) levels, yet found only a slight increase after $24 \mathrm{~h} \mathrm{(Fig.} \mathrm{5d).} \mathrm{Also,} \mathrm{on} \mathrm{a} \mathrm{cellular} \mathrm{level,} \mathrm{ROS}$ was not increased after $24 \mathrm{~h}$ (Additional file 4: Figure S4D). However, NADPH/NADP+ ratio was significantly decreased after $24 \mathrm{~h}$ hinting to a deregulation in cellular ROS (Fig. 5e), apparent at later timepoints. Deregulation in mitochondrial function, disruption of mitochondrial membrane potential and ROS generation are key players in induction of the mitochondrial apoptosis pathway. Indeed, we found an increase in cytosolic cytochrome C (Fig. 5f) as well as an increase in caspase 3 activation and Parp-1 cleavage after V-ATPase inhibition (Fig. 5g). Taken together, our data clearly show that lysosomal stress leads to alterations in LD and cardiolipin content subsequently an impairment of mitochondrial function, a reduction in energy generation and an induction of mitochondriadriven apoptosis (Fig. 5h).

\section{V-ATPase inhibition causes metabolic shift}

Since we discovered an increased glutamine dependency after V-ATPase inhibition (Fig. 5c), we were interested whether this can be exploited for novel therapeutic strategies. We used three different cell lines with varying Ras mutational status, as Ras mutations have been connected to mitochondrial function in the past $[29,30]$. First, we determined sensitivity to Arch in a proliferation assay, which showed that Ras wild-type cells (BxPC3 $\left.\mathrm{IC}_{50} 5.95 \mu \mathrm{M}\right)$ are less sensitive to the treatment than cells carrying Ras pathway mutations (Panc 03.27 $\mathrm{IC}_{50}$ $3.61 \mu \mathrm{M}, \mathrm{HUH7} \mathrm{IC}_{50} 2.83 \mu \mathrm{M}$ ) (Fig. 6a). We subsequently determined dose-response curves on proliferation inhibition in the presence or absence of the same metabolic inhibitors as used in the seahorse fuel flex test (Fig. 6b-d). This revealed that presence of BPTES, a glutaminase inhibitor, slightly decreased the $\mathrm{IC}_{50}$ values of Arch in all cell lines. Furthermore, inhibition of the glutamine pathway increased the capacity of Arch to induce apoptosis in all tested cell lines, with the greatest effect in Panc 03.27 cells (Fig. 6e-g). The potential to induce apoptosis is linked to the induction of a fission phenotype, as fission correlated with the ability to induce apoptosis (Fig. 6h).

\section{Discussion}

In this study, we unravel a novel role for lipid metabolism in cancer cells, linking lysosomal disturbance to an induction of mitochondrial apoptosis. We show that induction of lysosomal stress, especially by inhibition of the V-ATPase, leads to disturbance in cancer cell lipid content, which ultimately disrupts mitochondrial function.

The view of the lysosome has been changing dramatically in the recent past. Having simply been viewed as cellular trash can, the lysosome turns out to be a central regulator of cellular function and has been shown to be of particular importance in energy regulation [1]. Settembre and colleagues discovered that lysosomes are essential for maintaining cellular homeostasis, by sensing lysosomal nutrient status and inducing adaptations to starvation via a mechanism facilitating mTORC1 and transcription factor EB (TFEB). Upon lysosomal stress, TFEB translocates to the nucleus and induces lysosomal biogenesis [25]. Furthermore, the same group also discovered a role of TFEB in lipid regulation. They show that starvation induces TFEB translocation, which induces 


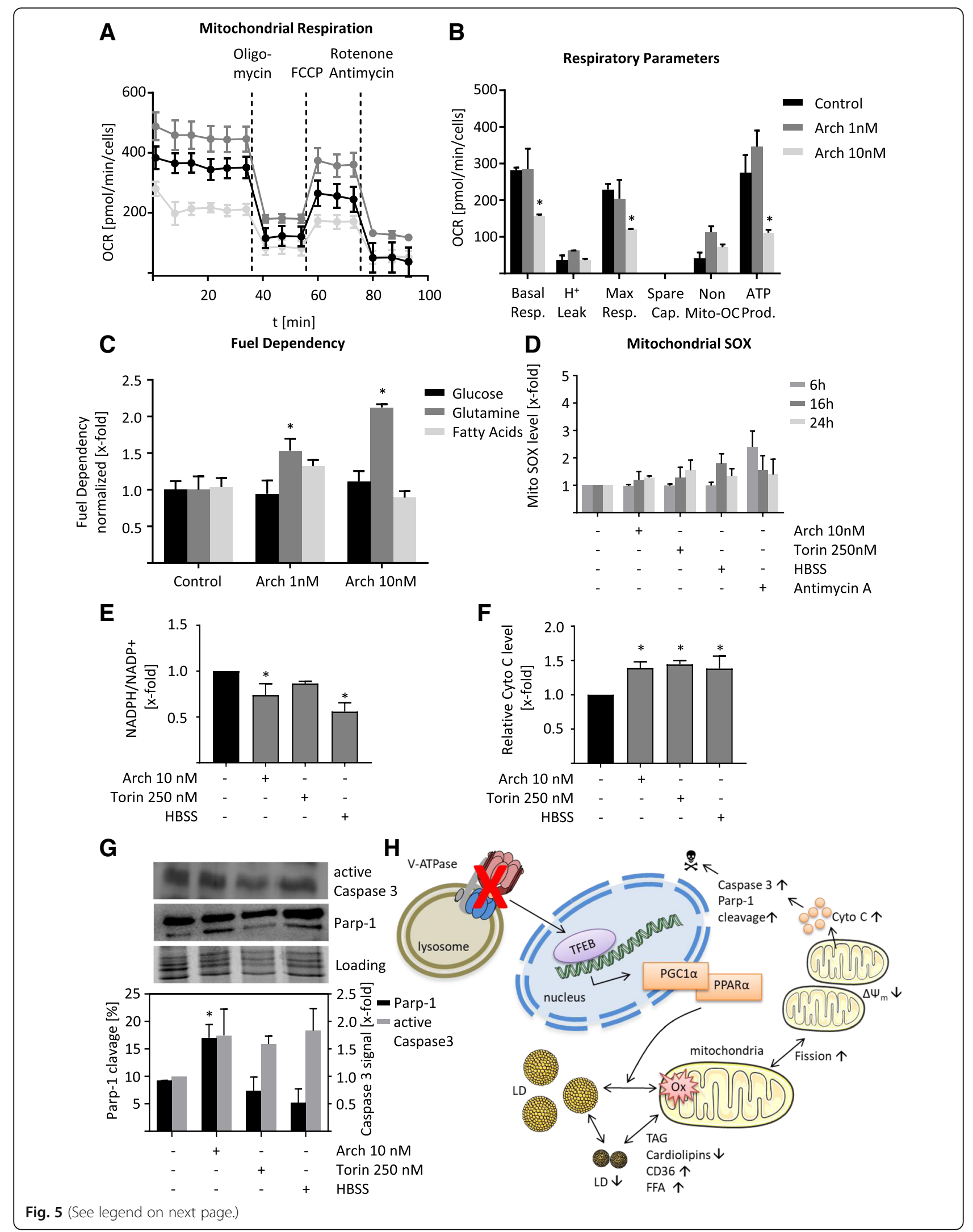


(See figure on previous page.)

Fig. 5 V-ATPase inhibition alters cellular metabolism. HUH7 cells were treated as indicated ( $24 \mathrm{~h}$ ). a Cells were exposed sequentially to oligomycin, FCCP and rotenone/antimycin. Vertical lines indicate time of addition of mitochondrial inhibitors. Oxygen consumption rate (OCR) was measured over time using a Seahorse XFe96 Analyzer. Cell mito stress test was performed according to manufacturer's protocol. b Respiratory parameters were calculated from OCR data (a) according to mitochondrial stress test protocol (User Guide Kit 103015-100 Agilent). c Mitochondrial fuel flex test was performed according to manufacturer's protocol (User Manual Kit 103270-100 Agilent). Fuel dependency was calculated as described in the manual. $\mathbf{d}$ Cells were loaded with MitoSOX ${ }^{\mathrm{TM}}$ and mitochondrial superoxide (SOX) was quantified by flow cytometry. e NADPH and NADP+ levels were assessed using NADP/NADPH-Glo ${ }^{\text {TM }}$ luminescence-based assay as described by the manufacturer (G9081 Promega). NADPH to NADP+ ratio was calculated and normalized to DMSO control. f Cytosolic cytochrome $\mathrm{C}$ was detected by flow cytometry. Bars are the mean + SEM of three independent experiments. $p^{*}<0.05$ (One-way ANOVA, Dunnett post test) (g) Protein levels of active Caspase 3 and Parp-1 cleavage have been determined and quantified by Western Blot. $\mathbf{h}$ Cartoon of mode of action. V-ATPase inhibition leads to transcriptional regulation of PGCla and PPAR a. Lipid droplets (LD) are changed in size and localization, leading to cardiolipin depletion, fission and impairment of mitochondrial function. This results in induction of mitochondria-driven apoptosis

global transcription control of lipid catabolism and lipophagy by induction of PGC1 $\alpha$ and PPAR $\alpha$ transcription [24]. Our data are in accordance with their findings, as we could also detect an induction of PGC1 $\alpha$ and PPAR $\alpha$. Yet, we suggest that this is not entirely mediated by TFEB translocation or translocation of TFE3 or MITF, two transcription factors of the same family that have also been connected to lysosome-to-nucleus signaling [31-33]. In our experiments, we could only detect a slight upregulation of nuclear translocation of TFEB, but not TFE3 or MITF, in HUH7 cells (Additional file 5: Figure S5A-C) using specific antibodies. However, we could confirm their data using constitutively active TFEB in HEK-293 cells (Additional file 5: Figure S5D). We hypothesize that upregulation of TFEB, PGC1 $\alpha$ and PPAR $\alpha$ as well as increasing lipid uptake via CD36 as discovered in our study is an escape mechanism, by which the cancer cells try to compensate for defective mitochondria and loss of energy induced by V-ATPase inhibition.

Mitochondrial function is crucially dependent on proper mitochondrial structure. This is especially true for the mitochondrial membrane. Mitochondria are double membrane bound organelles consisting of an inner and an outer membrane. The inner membrane displays a characteristic curvature that forms cristae, which are pivotal for mitochondrial respiration [16, 27]. A highly abundant lipid species of the mitochondrial inner membrane are cardiolipins, which are crucial for energy generation by oxidative phosphorylation [34]. Furthermore, mitochondria play a central role in regulating apoptosis. In their intermembrane space various proteins are stored, which can be released due to pro-apoptotic stimuli and activate caspase dependent cell death. Following proapoptotic stimuli, Bcl-2 family proteins Bak and Bax translocate to mitochondria and form a pore through which cytochrome $\mathrm{C}$ is released from the inter-membrane space into the cytosol to activate caspases [35]. In our study, we observed perturbations in lipid composition, in a manner that the level of saturated fatty acids increases, while the level of desaturated fatty acids decreases. Furthermore, we found a decrease in mitochondrial cardiolipins, which may lead to the observed mitochondrial dysfunction. While we were not able to detect significant translocation of Bak or Bax to the mitochondria (Additional file 4: Figure S4E), we observed cytochrome $C$ release, caspase activation and Parp cleavage, i.e. induction of apoptosis. Induction of apoptosis caused by saturated fatty acids has been observed also by others [36-38]. It was even reported that there is a differential role of saturated and unsaturated fatty acids, whereas unsaturated fatty acids influence autophagy but do not promote apoptosis, saturated fatty acids suppress autophagy and induce apoptosis [39]. However, other studies found that unsaturated lipids can induce apoptotic cell death, especially when peroxidized [40, 41]. We conclude that the observed changes in lipid composition caused by disruption of lysosomal function lead to a change in mitochondrial membrane composition, which on the one hand impairs mitochondrial function and on the other, triggers release of cytochrome $\mathrm{C}$ to the cytosol and subsequently induces apoptosis. This concept of inter-organelle cross talk between lysosomes and mitochondria is just emerging [15]. There is evidence that there exist direct contact sites between lysosomes and mitochondria in yeast, which facilitate the exchange of ions and phospholipids between the organelles [42, 43]. Yet, direct contact sites have only recently been discovered in skeletal muscle [44]. Interestingly, it has also been shown that lysosomal targeted formulations of 5-FU containing nanogels localize to the lysosomes and trigger mitochondria-driven apoptosis [45]. This phenomenon was induced by release of cathepsin B from lysosomes, which activated caspase 9, yet the arch induced mechanism cannot be identical, as our group could previously show that the level of active cathepsin $B$ is reduced after treatment [46]. Whether there exist direct contact sites in cancer cells or how these organelles exactly interact remains to be elucidated, however our data suggest that the two organelles strongly interact at least on a functional level. Additionally this study sheds more light on 

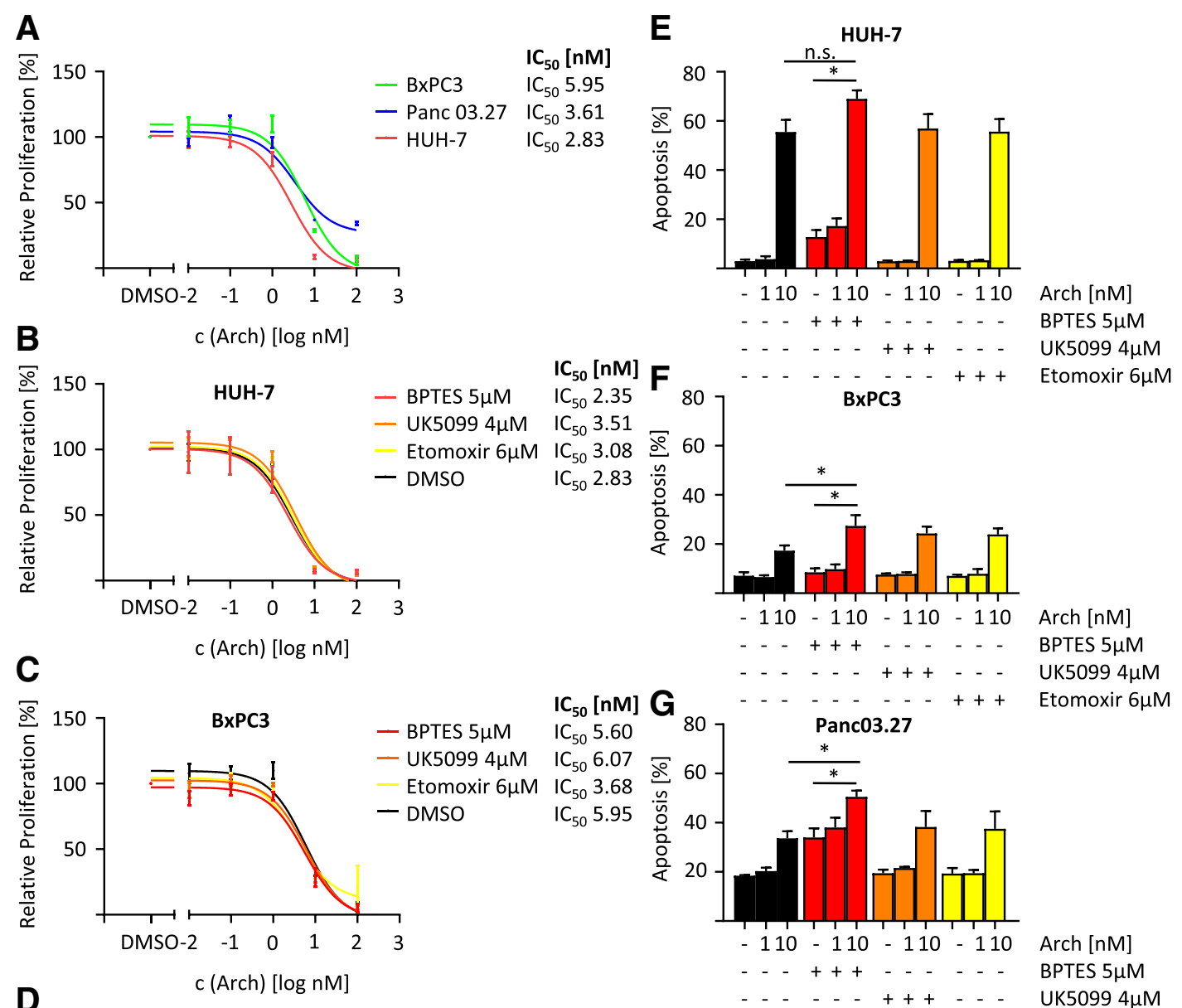

D.
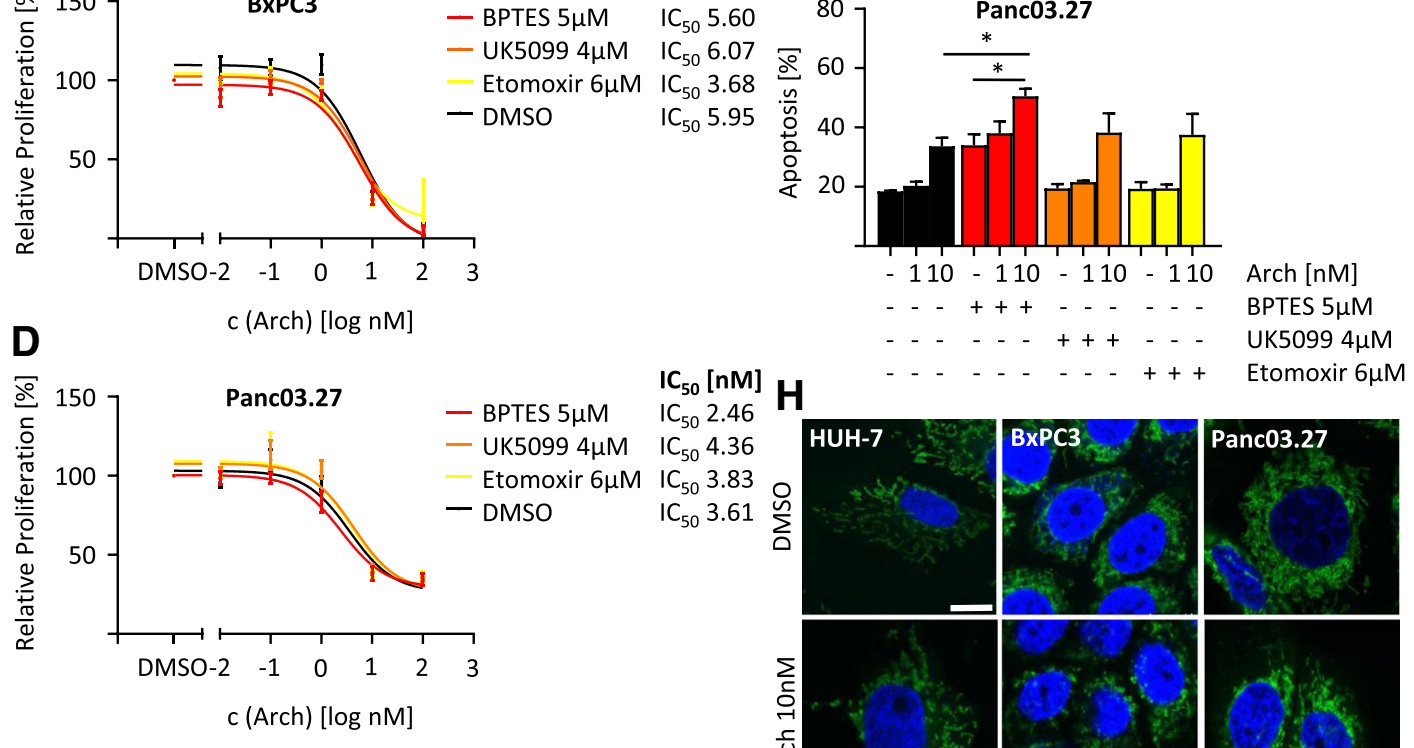

${ }^{\prime} C_{50} 2.46$

$\mathrm{IC}_{50} 4.36$

${ }^{I C} C_{50} 3.83$

$I_{50} 3.61 \sum^{n}$

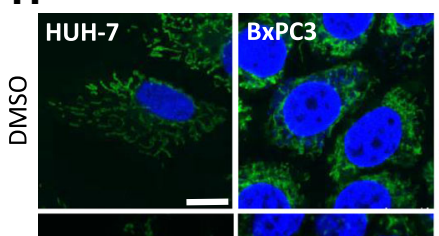

Panc03.27
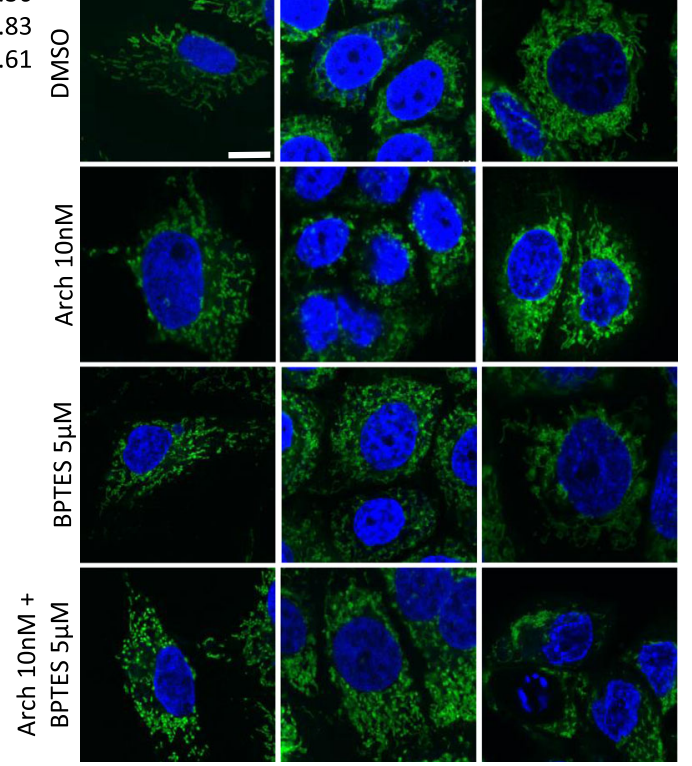

Fig. 6 (See legend on next page.) 
(See figure on previous page.)

Fig. 6 Glutamine deprivation sensitizes cancer cells towards V-ATPase inhibition. a Dose response curves for Arch treatment were determined after $72 \mathrm{~h}$. Dose response curves for Arch in the presence or absence (DMSO) of a constant concentration of BPTES $(5 \mu \mathrm{M})$ ), UK5099 (4 $\mu \mathrm{M})$ or etomoxir $(6 \mu \mathrm{M})$ of HUH7 (b), BxPC3 (c) and Panc03.27 (d) were determined after $72 \mathrm{~h}$ (a-d) Cell viability was analyzed by CellTiter Blue assay. IC 50 values were calculated by non-linear curve-fit using GraphPad Prism. HUH7 (e), BxPC3 (f) and Panc $03.27(\mathbf{g})$ were treated as indicated for $48 \mathrm{~h}$. Apoptotic cells were determined by Nicoletti assay. $\mathbf{h} \mathrm{HUH7}, \mathrm{BxPC} 3$ and Panc03.27 were treated as indicated for $24 \mathrm{~h}$, stained for mitochondria and nuclei and analyzed by confocal microscopy. Scale bar $10 \mu \mathrm{m}$. Values are the mean + SEM of three independent experiments. $p^{*}<0.05$ (Oneway ANOVA, Tukey post test)

the mechanism by which V-ATPase inhibition leads to mitochondria-driven apoptosis, which our group already investigated in the past [10].

Interestingly, oncogenic Ras signaling has been connected to mitochondrial function. Ras is a wellestablished oncogene, which is hyperactive in a variety of cancers $[47,48]$. Ras exists in three isoforms, namely K-Ras, H-Ras and N-Ras, all of which display oncogenic potential. Additionally also Ras pathway mutations, downstream of Ras itself have been linked to cancer progression [49]. The exact mechanisms of Ras-induced malignant transformation are currently still investigated. $\mathrm{Hu}$ et al. could show that oncogenic K-Ras is associated with mitochondria where it changes the metabolic phenotype of the cells promoting Warburg effect [50] and drives tumor development [51]. Serasinghe and colleagues provide data supporting the findings of $\mathrm{Hu}$ et al. They show that an oncogenic Ras mutation is closely linked to changes in mitochondria. In their study they found that mitochondrial fission by Drp1 is essential for Ras malignant transformation and that also constitutively active MAPK signaling downstream of Ras induces mitochondrial changes in a Drp1 dependent manner [29]. As our previous work showed that inhibition of the V-ATPase is able to inhibit Ras pathway activation [9] and our current work connects lysosomes and mitochondria we assumed a possibility to address Ras mutated cells by manipulation of lysosomal function. Indeed, we found that K-Ras mutated Panc 03.27 cells and HUH7, which also show constitutive Ras pathway activation, are more sensitive to V-ATPase inhibition than the Ras wild-type BxPC3. These findings provide a first hint that Ras pathway mutations might sensitize cells for treatment with V-ATPase inhibitors, a connection that certainly should be addressed with future research.

\section{Conclusion}

The present work provides a novel role for cellular lipid metabolism in the organelle cross-talk between lysosomes and mitochondria. Induction of lysosomal stress, especially by inhibiting the V-ATPase, leads to a reduction in mitochondrial cardiolipin content, induces fission, disrupts mitochondrial function and induces mitochondria-driven apoptosis. The study enhances our understanding of the interaction between lysosomes and mitochondria as well as the mechanism by which V-ATPase inhibition induces apoptosis. This increase in knowledge will help to develop new anti-cancer therapeutic strategies targeting the lysosome-mitochondria axis.

\section{Additional files}

Additional file 1: Figure S1. (A) Cells were treated as indicated for $24 \mathrm{~h}$. Lipids from whole cells (HUH7, HepG2 and Hep3B) were isolated. Total amounts of TAG were determined by UPLC-MS/MS and normalized to cell numbers. Bars are the mean+SEM of three independent experiments. $p^{*}<0.05$ (paired student t-test). Cells were treated with vehicle (DMSO) as indicated (24 h). Lipids from whole cells lysates of HUH7 (B), HepG2 (C), and Hep3B (D) were isolated, and the cellular proportion of TAG species was analyzed by UPLC-MS/MS. Lipids from isolated lysosomes (E) or mitochondria (F) were isolated. Total amounts of TAG were determined by UPLC-MS/MS and normalized to cell numbers. Bars are the mean+SEM of three independent experiments. $\mathrm{p}^{*}<0.05$ (paired student t-test). Lipids from isolated lysosomes $(\mathrm{G})$ and mitochondria $(H)$ of $H U H 7$ were isolated, and the cellular proportion of TAG species was analyzed by UPLC-MS/MS. Data are given as percentage of all TAG species detected (100\%). Bars are the mean+SEM of three independent experiments. (PDF $196 \mathrm{~kb}$ )

Additional file 2: Figure S2. (A-C) HUH-7 cells were treated as indicated. Relative mRNA expression levels of NRF1 (A), NRF2 (B) and ERRa (C) were detected by GPCR. (D, E) Cells were labeled with Bodipy 558/568 Red C-12 (cyan) Bodipy 493/503 (magenta) and lysotracker (D) (yellow) or mitotracker (E) (yellow), repectively. Scale bar $25 \mu \mathrm{m}$. Representative images out of three independent experiments are shown. (PDF $441 \mathrm{~kb}$ )

Additional file 3: Figure S3. $\mathrm{HUH}-7$ cells were treated as indicated. Representative images out of three independent experiments are shown. (A) Protein level was detected by WB. Total protein served as loading control. Quantification of Drp1 phosphorylation (bar graphs). (B) Confocal live cell imaging was performed staining for mitochondria (green), lysosomes (red) and nuclei (blue). Scale bar $25 \mu \mathrm{m}$. (PDF 574 kb)

Additional file 4: Figure S4. HUH-7 cells were treated as indicated. Representative images out of three independent experiments are shown. (A) Protein expression ( $24 \mathrm{~h}$ ) of ACADVL, ACADM and HADHA was detected by WB. Total protein served as loading control. (B) Relative ATP levels were assessed by CellTiter-Glo ${ }^{\oplus}$ assay according to manufacturer's protocol after $24 \mathrm{~h}$ treatment as indicated. (C) Mitochondrial Fuel Flex Test was performed according to manufacturer's protocol (User Manual Kit 103270-100 Agilent) and capacity was calculated as described in the manual. (D) Cells were loaded with the redox sensitive dye Carboxy$\mathrm{H}_{2}$ DCFDA (DCF) and analyzed by flow cytometry. Quantification of DCF fluorescence intensity normalized to DMSO control. Bars are the SEM of three independent experiments. (E) Protein expression (24 h) of Bax and Bak was detected by WB on isolated mitochondria. Total protein served as loading control. (PDF $472 \mathrm{~kb}$ ) 
Additional file 5: Figure S5. HUH-7 cells were treated as indicated. Representative images out of three independent experiments are shown. (A) Cells were fixed and stained for TFE3, MITF or TFEB, respectively (green) and nuclei (blue) and analyzed by confocal microscopy. Scale bar $10 \mu \mathrm{m}$. (B) Cell lysates were fractioned in nuclei and cytosolic fractions and was protein expression of TFE3, MITF and TFEB, was detected by WB. Total protein served as loading control. Nuclear levels of transcription factors were quantified (bar graphs) (C) Relative mRNA expression levels of TFEB, TFE3 and MITF were detected by GPCR. (D) Wilde-type TFEB and a consitutively active TFEB mutant (TFEB) tagged with GFP (green) were overexpressed in HEK 293 cells. Cells were fixed, co-stained for nuclei and analyzed by confocal microscopy. Scale bar $7.5 \mu \mathrm{m}$. (PDF $673 \mathrm{~kb}$ )

\section{Abbreviations}

EM: Electron microscopy; HBSS: Hank's balanced salt solution; HCC: hepatocellular carcinoma LDlipid droplet; MAPK: Mitogen-activated protein kinase; ROS: Reactive oxygen species; TAG: Triacylglcerid; VATPase: Vacuolar $\mathrm{H}^{+}$-ATPase; WB: Western Blot

\section{Acknowledgements}

We thank Bernadette Grohs for the excellent technical assistance.

\section{Authors' contributions}

KB: conception and design, development of methodology, acquisition, analysis and interpretation of data, writing of the manuscript; HP, AK, OW: acquisition, analysis and interpretation of TAG and acyl-CoA data; BP, SS, HZ: acquisition and interpretation of electron microscopy data; SJ, AS: conception and interpretation of Seahorse experiments; FL: acquisition and analysis of proliferation and apoptosis data; RM: material support, review of the manuscript; AV: conception and design of the work, final approval of published article version, study supervision; KS: conception and design of the work, study supervision, interpretation of data. All authors read and approved the final manuscript.

\section{Authors' information}

Not applicable

\section{Funding}

This work was supported by the DFG research funds 1406 SCHW 1781/1-1 and AV 376/18-1.

\section{Availability of data and materials}

All data generated or analyzed during this study are included in this published article and its supplementary information files.

\section{Ethics approval and consent to participate}

Not applicable

\section{Consent for publication}

Not applicable

\section{Competing interests}

The authors declare that they have no competing interests.

\section{Author details}

'Department of Pharmacy, Pharmaceutical Biology,

Ludwig-Maximilians-Universität München, Butenandtstr. 5-13, 81377 Munich, Germany. ${ }^{2}$ Chair of Pharmaceutical/Medicinal Chemistry, Institute of Pharmacy, Friedrich Schiller University Jena, Philosophenweg 14, 07743 Jena, Germany. ${ }^{3}$ Department of Anatomy and Cell Biology, Biomedical Center, Ludwig-Maximilians-Universität München, Grosshaderner Strasse 9, 82152 Planegg-Martinsried, Germany. ${ }^{4}$ Institute of Toxicology and Environmental Hygiene, Technical University Munich, School of Medicine, 80802 Munich, Germany. ${ }^{5}$ Department of Biochemistry and Molecular Biology, Theodor-Boveri-Institute, Biocenter, Am Hubland, 97074 Würzburg, Germany. ${ }^{6}$ Institute of Molecular Toxicology and Pharmacology, Helmholtz Center Munich, German Research Center for Environmental Health, 85764 Neuherberg, Germany. ${ }^{7}$ Helmholtz Centre for Infection Research and Department of Pharmaceutical Biotechnology, Helmholtz Institute for
Pharmaceutical Research Saarland, Saarland University, PO 151150, Universitätscampus E8 1, 66123 Saarbrücken, Germany.

Received: 16 May 2019 Accepted: 15 July 2019

Published online: 29 July 2019

\section{References}

1. Settembre C, Fraldi A, Medina DL, Ballabio A. Signals from the lysosome: a control Centre for cellular clearance and energy metabolism. Nat Rev Mol Cell Biol. 2013;14(5):283-96.

2. Saftig P, Klumperman J. Lysosome biogenesis and lysosomal membrane proteins: trafficking meets function. Nat Rev Mol Cell Biol. 2009;10(9):623-35.

3. Polishchuk EV, Merolla A, Lichtmannegger J, Romano A, Indrieri A, llyechova EY, et al. Activation of Autophagy, Observed in Liver Tissues From Patients With Wilson Disease and From ATP7B-Deficient Animals, Protects Hepatocytes From Copper-Induced Apoptosis. Gastroenterology. 2019;156(4):1173-89.e5.

4. Piao S, Amaravadi RK. Targeting the lysosome in cancer. Ann N Y Acad Sci. 2016:1371(1):45-54.

5. Santos CR, Schulze A. Lipid metabolism in cancer. FEBS J. 2012;279(15):2610-23.

6. Rohrig F, Schulze A. The multifaceted roles of fatty acid synthesis in cancer. Nat Rev Cancer. 2016;16(11):732-49.

7. Long J, Zhang C-J, Zhu N, Du K, Yin Y-F, Tan X, et al. Lipid metabolism and carcinogenesis, cancer development. Am J Cancer Res. 2018;8(5):778-91.

8. Zhang F, Du G. Dysregulated lipid metabolism in cancer. World J Biol Chem. 2012;3(8):167-74.

9. Bartel K, Winzi M, Ulrich M, Koeberle A, Menche D, Werz O, et al. VATPase inhibition increases cancer cell stiffness and blocks membrane related Ras signaling - a new option for HCC therapy. Oncotarget. 2017 8(6):9476-87.

10. von Schwarzenberg K, Wiedmann RM, Oak P, Schulz S, Zischka H, Wanner G, et al. Mode of cell death induction by pharmacological vacuolar H+-ATPase (V-ATPase) inhibition. J Biol Chem. 2013;288(2):1385-96.

11. McHenry P, Wang WL, Devitt E, Kluesner N, Davisson VJ, McKee E, et al. lejimalides a and B inhibit lysosomal vacuolar H+-ATPase (V-ATPase) activity and induce S-phase arrest and apoptosis in MCF-7 cells. J Cell Biochem. 2010;109(4):634-42.

12. Teplova W, Tonshin AA, Grigoriev PA, Saris NE, Salkinoja-Salonen MS Bafilomycin $A 1$ is a potassium ionophore that impairs mitochondrial functions. J Bioenerg Biomembr. 2007;39(4):321-9.

13. Aiko K, Tsujisawa T, Koseki T, Hashimoto S, Morimoto Y, Amagasa T, et al. Involvement of cytochrome $\mathrm{c}$ and caspases in apoptotic cell death of human submandibular gland ductal cells induced by concanamycin a. Cell Signal. 2002;14(8):717-22.

14. Murley A, Nunnari J. The emerging network of mitochondria-organelle contacts. Mol Cell. 2016;61(5):648-53.

15. Todkar K, llamathi HS, Germain M. Mitochondria and Lysosomes: Discovering Bonds. Front Cell Dev Biol. 2017;5(106):106. https://doi.org/10.33 89/fcell.2017.00106.

16. Twig G, Hyde B, Shirihai OS. Mitochondrial fusion, fission and autophagy as a quality control axis: the bioenergetic view. Biochim Biophys Acta Bioenerg. 2008;1777(9):1092-7.

17. Schieder M, Rotzer K, Bruggemann A, Biel M, Wahl-Schott C. Planar patch clamp approach to characterize ionic currents from intact lysosomes. Sci Signal. 2010;3(151):pl3.

18. Abcam. Subcellular fractionation protocol [Available from: https://www. abcam.com/protocols/subcellular-fractionation-protocol.

19. Koeberle A, Pergola C, Shindou H, Koeberle SC, Shimizu T, Laufer SA, et al. Role of p38 mitogen-activated protein kinase in linking stearoyl-CoA desaturase-1 activity with endoplasmic reticulum homeostasis. FASEB J. 2015;29(6):2439-49.

20. Glatzel DK, Koeberle A, Pein H, Loser K, Stark A, Keksel N, et al. Acetyl-CoA carboxylase 1 regulates endothelial cell migration by shifting the phospholipid composition. J Lipid Res. 2018;59(2):298-311.

21. Wiedmann RM, von Schwarzenberg K, Palamidessi A, Schreiner $L$, Kubisch $R$, Liebl J, et al. The V-ATPase-inhibitor archazolid abrogates tumor metastasis via inhibition of endocytic activation of the rho-GTPase Rac1. Cancer Res. 2012;72(22):5976-87.

22. Schneider LS, von Schwarzenberg K, Lehr T, Ulrich M, Kubisch-Dohmen R, Liebl J, et al. Vacuolar-ATPase inhibition blocks Iron metabolism to mediate therapeutic effects in breast Cancer. Cancer Res. 2015;75(14):2863-74. 
23. Schempp CM, von Schwarzenberg K, Schreiner L, Kubisch R, Muller R,

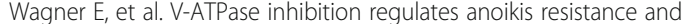
metastasis of cancer cells. Mol Cancer Ther. 2014;13(4):926-37.

24. Settembre C, De Cegli R, Mansueto G, Saha PK, Vetrini F, Visvikis O, et al. TFEB controls cellular lipid metabolism through a starvation-induced autoregulatory loop. Nat Cell Biol. 2013;15(6):647-58.

25. Settembre C, Zoncu R, Medina DL, Vetrini F, Erdin S, Erdin S, et al. A lysosome-to-nucleus signalling mechanism senses and regulates the lysosome via mTOR and TFEB. EMBO J. 2012;31(5):1095-108.

26. Zoncu R, Bar-Peled L, Efeyan A, Wang S, Sancak Y, Sabatini DM. mTORC1 senses lysosomal amino acids through an inside-out mechanism that requires the vacuolar H(+)-ATPase. Science. 2011;334(6056):678-83.

27. Chicco AJ, Sparagna GC. Role of cardiolipin alterations in mitochondrial dysfunction and disease. Am J Physiol Cell Physiol. 2007;292.

28. Gomes LC, Scorrano L. Mitochondrial morphology in mitophagy and macroautophagy. Biochim Biophys Acta Mol Cell Res. 2013;1833(1):205-12.

29. Serasinghe Madhavika N, Wieder Shira Y, Renault Thibaud T, Elkholi R, Asciolla James J, Yao Jonathon $L$, et al. Mitochondrial division is requisite to RAS-induced transformation and targeted by oncogenic MAPK pathway inhibitors. Mol Cell. 2015;57(3):521-36.

30. Weinberg F, Hamanaka R, Wheaton WW, Weinberg S, Joseph J, Lopez M, et al. Mitochondrial metabolism and ROS generation are essential for Krasmediated tumorigenicity. Proc Natl Acad Sci. 2010;107(19):8788-93.

31. Martina JA, Diab HI, Brady OA, Puertollano R. TFEB and TFE3 are novel components of the integrated stress response. EMBO J. 2016;35(5):479-95.

32. Raben N, Puertollano R. TFEB and TFE3: linking lysosomes to cellular adaptation to stress. Annu Rev Cell Dev Biol. 2016;32(1):255-78.

33. Magers MJ, Udager AM, Mehra R. MiT family translocation-associated renal cell carcinoma: a contemporary update with emphasis on morphologic, Immunophenotypic, and molecular mimics. Arch Pathol Lab Med. 2015;139(10):1224-33.

34. Schug ZT, Gottlieb E. Cardiolipin acts as a mitochondrial signalling platform to launch apoptosis. Biochim Biophys Acta. 2009;1788.

35. Wang C, Youle RJ. The role of mitochondria in apoptosis. Annu Rev Genet. 2009:43(1):95-118.

36. Roongta UV, Pabalan JG, Wang X, Ryseck RP, Fargnoli J, Henley BJ. Cancer cell dependence on unsaturated fatty acids implicates stearoyl-CoA desaturase as a target for cancer therapy. Mol Cancer Res. 2011;9.

37. Hardy S, El-Assaad W, Przybytkowski E, Joly E, Prentki M, Langelier Y. Saturated fatty acid-induced apoptosis in MDA-MB-231 breast cancer cells. A role for cardiolipin. J Biol Chem. 2003:278.

38. Nolan CJ, Larter CZ. Lipotoxicity: why do saturated fatty acids cause and monounsaturates protect against it? J Gastroenterol Hepatol. 2009;24(5):703-6.

39. Mei S, Ni HM, Manley S, Bockus A, Kassel KM, Luyendyk JP, et al. Differential roles of unsaturated and saturated fatty acids on autophagy and apoptosis in hepatocytes. J Pharmacol Exp Ther. 2011;339(2):487-98.

40. Ayala A, Munoz MF, Argguelles S. Lipid peroxidation: production, metabolism, and signaling mechanisms of malondialdehyde and 4-Hydroxy2-Nonenal. Oxidative Med Cell Longev. 2014;2014:31.

41. Iuchi K, Ema M, Suzuki M, Yokoyama C, Hisatomi H. Oxidized unsaturated fatty acids induce apoptotic cell death in cultured cells. Mol Med Rep. 2019;19(4):2767-73.

42. Elbaz-Alon Y, Rosenfeld-Gur E, Shinder V, Futerman Anthony H, Geiger T, Schuldiner M. A dynamic Interface between vacuoles and mitochondria in yeast. Dev Cell. 2014;30(1):95-102.

43. Hönscher C, Mari M, Auffarth K, Bohnert M, Griffith J, Geerts W, et al. Cellular metabolism regulates contact sites between vacuoles and mitochondria. Dev Cell. 2014;30(1):86-94.

44. Aston D, Capel RA, Ford KL, Christian HC, Mirams GR, Rog-Zielinska EA, et al. High resolution structural evidence suggests the sarcoplasmic reticulum forms microdomains with acidic stores (lysosomes) in the heart. Sci Rep. 2017;7:40620.

45. Zhu X, et al. Mastocarcinoma therapy synergistically promoted by lysosome dependent apoptosis specifically evoked by 5-Fu@nanogel system with passive targeting and $\mathrm{pH}$ activatable dual function. J Control Release. 2017;254:107-18.

46. Kubisch R, Frohlich T, Arnold GJ, Schreiner L, von Schwarzenberg K, Roidl A, et al. V-ATPase inhibition by archazolid leads to lysosomal dysfunction resulting in impaired cathepsin B activation in vivo. Int J Cancer. 2014; 134(10):2478-88.

47. Downward J. Targeting RAS signalling pathways in cancer therapy. Nat Rev Cancer. 2003;3(1):11-22.
48. Whittaker S, Marais R, Zhu AX. The role of signaling pathways in the development and treatment of hepatocellular carcinoma. Oncogene. 2010;29(36):4989-5005.

49. Llovet JM, Bruix J. Molecular targeted therapies in hepatocellular carcinoma. Hepatology. 2008;48(4):1312-27.

50. Hsu PP, Sabatini DM. Cancer cell metabolism: Warburg and beyond. Cell. 2008;134(5):703-7

51. Hu Y, Lu W, Chen G, Wang P, Chen Z, Zhou Y, et al. K-ras(G12V) transformation leads to mitochondrial dysfunction and a metabolic switch from oxidative phosphorylation to glycolysis. Cell Res. 2012;22(2):399-412.

\section{Publisher's Note}

Springer Nature remains neutral with regard to jurisdictional claims in published maps and institutional affiliations.

\section{Ready to submit your research? Choose BMC and benefit from:}

- fast, convenient online submission

- thorough peer review by experienced researchers in your field

- rapid publication on acceptance

- support for research data, including large and complex data types

- gold Open Access which fosters wider collaboration and increased citations

- maximum visibility for your research: over $100 \mathrm{M}$ website views per year

At BMC, research is always in progress.

Learn more biomedcentral.com/submissions 